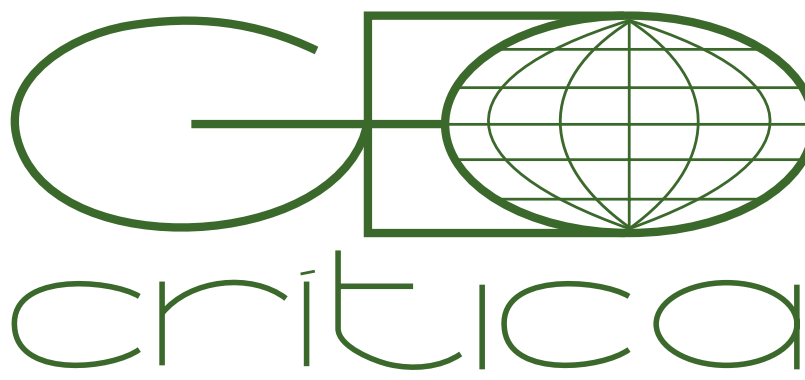

\section{Scripta Nova}

Revista Electrónica de Geografía y Ciencias Sociales Universitat de Barcelona

15 de Marzo de 2020

\title{
LA ADAPTACIÓN COMO RESPUESTA AL CAMBIO CLIMÁTICO. NOTAS ACERCA DE LAS CONTRACARAS DE UN PARADIGMA DOMINANTE
}

\author{
Paula Mussetta \\ Universidad Nacional de Cuyo, Argentina. \\ pcmussetta@gmail.com
}

Recibido: 4 febrero 2019; Devuelto para correcciones: 12 mayo 2019; Aceptado: 7 julio 2019

\begin{abstract}
La adaptación como respuesta al cambio climático. Notas a cerca de las contracaras de un paradigma dominante. (Resumen)

La adaptación es una propuesta que ha generado gran atención para afrontar el cambio climático en el último tiempo. En la versión dominante que circula por la academia y gobiernos ella es presentada como una respuesta necesaria a las nuevas y cambiantes condiciones del entorno. Esa visión es problemática porque se ocupa más de los efectos que de las causas de la crisis climática, pero principalmente porque evade el contenido estrictamente político y económico de los problemas alrededor de la misma. Siguiendo autores de la ecología política el artículo cuestiona su potencial como respuesta a la crisis climática. Concluye que las salidas genuinas a la crisis provendrán de enfoques que sitúen en el centro del análisis la relación entre cambio climático y modelo de producción de la humanidad y la naturaleza, aspecto que no tiene cabida en un marco como el de la adaptación.
\end{abstract}

Palabras clave: adaptación, cambio climático, ecología política, producción social de la naturaleza, crisis del modelo civilizatorio

Adaptation as a response to climate change. Notes on the downsides of a mainstream paradigm. (Abstract)

Adaptation is a proposal that has generated a great deal of attention addressing climate change. In the dominant version circulating through academia and governments, it is presented as a essential response to new and changing environmental conditions. This view is problematic because it deals more with the effects than with the causes of the climate crisis and evades the strictly political and economic content of the problems around them. Following political ecology authors, the article questions its potential as a profound response to the climate crisis. The article concludes that genuine solutions to the crisis will come from approaches that place at the core of the analysis the relationship between climate change and the production model of humanity and nature at the centre of the scene, an aspect that has no place in a framework such as that of adaptation.

Keywords: adaptation, climate change, political ecology, social production of nature , civilizing model crisis 
El cambio climático es parte de una crisis socioecológica más amplia marcada por la desmedida explotación de recursos naturales y un determinado patrón de uso de combustibles fósiles. No se trata de una amenaza para futuras generaciones sino que se ha posicionado como uno de los problemas que definen nuestra contemporaneidad. Por lo tanto, constituye un tema central para la investigación y el diseño de políticas públicas en distintos niveles de gobierno y pone en marcha una maquinaria que opera en esa doble dirección. De hecho es un campo desde el que emergen una serie de conceptos -como el de servicios ecosistémicos, vulnerabilidad, resiliencia, capacidad adaptativa- que operan en la frontera entre la gestión y la ciencia.

La relación entre política y ciencia en torno al cambio climático es estrecha y se retroalimenta circulando entre uno y otro ámbito. En la política, los organismos internacionales, algunos creados especialmente a raíz de este asunto (como el Grupo Intergubernamental de Expertos sobre el Cambio Climático -IPCC-, la Convención Marco de las Naciones Unidas sobre el Cambio Climático y sus periódicas Conferencias de la Partes -COP-, por citar sólo dos de los más notorios) destinan enormes montos de dinero para financiar investigación básica y aplicada a fin de medir y revertir los procesos vinculados al cambio climático. Son estos actores de la gobernanza climática los encargados por un lado, de pensar y diseñar las alternativas de salida y transición a la crisis que este fenómeno nos impone. Por otro lado, a partir de una densa arquitectura programática se esfuerzan por trascender la escala global desde la que estudian y construyen el problema para penetrar en escalas nacionales, regionales y hasta locales. Llevan así hasta los lugares más remotos del planeta las respuestas pensadas en estos think tanks globales. A lo largo de un entramado de instituciones y relaciones que articulan expertos, procesos y discursos a diferentes escalas se produce y circula lo que Ulloa llama ecogubernamentalidad climática: un discurso científico climático y ambiental dominante que tiene una alta incidencia en la formulación de políticas locales. ${ }^{1}$

Esta gobernanza climática global tiene a su vez un sólido punto de apoyo en el ámbito académico. Allí se produce, predominantemente desde las ciencias naturales y físicas, la "ciencia del cambio climático". Esta corriente dominante entiende que el cambio climático es un cambio de clima atribuido directa o indirectamente a la actividad humana que altera la composición de la atmósfera mundial. ${ }^{2}$ Estas alteraciones ambientales exógenas por actividades humanas externas, impactan en la esfera social de una manera objetiva; esto es, sin considerar en principio mediaciones sociales, espaciales, históricas, etc. La incidencia de las ciencias sociales es más reciente y aún no tiene fuerza para constituirse en un enfoque que no sea subsidiario de las primeras. Los grandes desarrolladores de la ciencia del cambio climático producen y expanden esta visión dominante sobre el cambio climático que es la globalmente legítima, aceptada y reconocida. ${ }^{3}$ Esta perspectiva es adoptada por los organismos

1 Ulloa, 2016.

2 UN, 1992.

3 Howe, 2014. 
internacionales quienes a su vez producen las grandes opciones o alternativas para afrontar la crisis climática y sus impactos.

El abanico de opciones, alternativas y respuestas concretas que se proponen derivadas de esa corriente principal también son planteadas como necesarias, urgentes y como veremos más adelante, naturales. A la vez, son esgrimidas como tecno-científicas y con ello se auto-postulan como a-políticas. No tienen en cuenta el hecho de que la ciencia (los enfoques, las teorías, los conceptos) no es ni social ni políticamente neutral sino que está indisolublemente asociada a diferentes posicionamientos políticos. Como sostiene Lampis, la ciencia en tanto comprensión de la realidad hace de las definiciones un punto álgido en el proceso de intercambio entre ciencia y política pública, claramente central en el caso del cambio climático. ${ }^{4}$ De hecho, definir de una manera u otra un fenómeno implica priorizar cierto tipo de conocimiento sobre otros y condicionar las respuestas al fenómeno. Sin embargo, existe una tendencia general a no explicitar los supuestos o implicaciones de los enfoques y adoptar conceptos y técnicas sin reparar los contextos en los que fueron desarrollados.

Dos de las principales respuestas de esta corriente principal para afrontar el problema son la mitigación y la adaptación. La primera marcó el rumbo de las políticas desde la la Conferencia de Río de Janeiro sobre Medio Ambiente y Desarrollo en 1992 hasta la publicación del Cuarto Reporte del IPCC. ${ }^{5}$ Se trataba básicamente de intervenciones para reducir las fuentes de creación o para mejorar los sumideros de los gases que causan el calentamiento global. Por ejemplo, el uso tecnologías generadoras de energías limpias no contaminantes. La mitigación representa una comprensión parcial del problema porque pondera las dimensiones biofísicas del cambio climático. Además, ante la constatación de que la concentración de gases parecía no ceder a las medidas aplicadas la adaptación se fue consolidando como una alternativa superadora de la mitigación al relacionar el cambio climático con problemas sociales y económicos. Su formalización se produce en 2006 en la Cumbre de Nairobi. ${ }^{6}$ Hoy mitigación y adaptación coexisten y se complementan pero cada una se orienta a objetivos distintos. Sin embargo, la adaptación es actualmente unos de los principales ejes articuladores de las respuestas del cambio climático.

La adaptación no es sólo una herramienta técnica sino que es a la vez un paradigma en el más clásico sentido kuhniano del término: una matriz disciplinar que constituye un patrón a seguir en el que convergen las miradas de un conjunto de científicos. La adaptación es un paradigma porque es una propuesta que reúne un conjunto de ideas, conceptos y postulados que no sólo interpretan la realidad sino que buscan incidir en ella; guía la investigación y la política para afrontar la crisis climática y sus impactos. La corriente principal de la adaptación que circula por academia y gobiernos se deriva de la versión dominante del cambio climático y se

\footnotetext{
4 Lampis, 2012.

5 Palacio, 2017.

6 Taylor, 2014.
} 
ocupa más de los efectos que de las causas del problema. De este modo, evade el contenido estrictamente político e histórico de los problemas alrededor de la misma. Frente a ello, partimos de la idea de que la adaptación tiene un fundamento político que es naturalizado dentro de su marco discursivo y está orientado a sostener el modelo de producción y acumulación vigente. Las respuestas dadas desde ese paradigma lejos de favorecer el bienestar humano o la justicia climática y ambiental tienden a sostener el statu quo que origina la crisis y las desigualdades sociales en torno a ella. Este es el punto de partida del análisis que sigue.

A partir de una estrategia metodológica de selección, revisión y análisis bibliográfico, este artículo presenta un recorrido principalmente conceptual que propone una relectura de la adaptación a la luz de algunos autores que han cuestionado este enfoque. El artículo indaga los supuestos del paradigma de la adaptación y explora algunos de sus problemáticos alcances e implicaciones. La reconstrucción sintetiza los aportes desde la ecología política y otros autores del pensamiento ambiental crítico. Luego de esta explicitación de postulados e implicaciones se plantea si es posible una reconceptualización crítica de la adaptación que supere las debilidades señaladas en su versión dominante y desde la cual elaborar propuestas de fondo para afrontar el cambio climático. El artículo concluye señalando la dificultad de conciliar la adaptación con un abordaje que cuestione la causas de la crisis climática. Por lo tanto, remarca la necesidad de ceder el protagonismo de la adaptación hacia otros marcos y paradigmas desde los cuales pensar alternativas a la crisis climática así como contribuir a resolver sus causas profundas.

El trabajo está organizado de la siguiente manera. La primera sección, describe brevemente de qué trata la propuesta de la adaptación y por qué ha resultado una corriente principal exitosa. La segunda remite a los orígenes de la adaptación en el paradigma funcionalista y de la ecología cultural. Seguidamente, desarrolla la reconceptualización de la adaptación desde sus bases originarias al cambio climático.

A continuación se repasan algunos intentos que han intentado superar la estrechez del análisis propio de la corriente principal. Se argumenta que si bien la adaptación ha sido reconceptualizada dentro del marco del cambio climático, los defectos de los supuestos originales permanecen: el carácter biologicista y mecanicista de las relaciones sociedad-naturaleza eluden la incorporación de las dimensiones inherentemente políticas. El apartado que sigue desarrolla uno de los más importantes supuestos que la adaptación reproduce: la dicotomía ontológica entre clima/naturaleza y sociedad. Este supuesto refuerza el blindaje del pensamiento adaptativo al reconocimiento de la producción social de la naturaleza. El último apartado reconstruye cómo el paradigma de la adaptación, junto al de la resiliencia, despliegan un tipo de racionalidad calculativa que tiende a una visión privada del riesgo propia del neoliberalismo. En las conclusiones se argumenta por qué es necesario superar el paradigma de la adaptación y concentrarse en otras miradas, otros enfoques que entiendan que el cambio climático es producto de una crisis de la producción de 
la humanidad y de la naturaleza, una crisis civilizatoria. Sólo desde allí será posible pensar alternativas a la crisis socioecológica que hoy afecta a la humanidad.

La crítica académica a la crisis ecopolítica es amplia y tiene además una nutrida base en centros académicos latinoamericanos. No obstante, son escasos los trabajos que focalizan la mirada sobre las propuestas de solución que aportan las corrientes principales del cambio climático. Este artículo se inserta en estos debates y pretende abonar desde allí a la crítica.

\section{El auge de la adaptación como respuesta al cambio climático.}

El Tercer Reporte del IPCC planteó en 2001 que la adaptación al cambio climático es el proceso de ajuste de los sistemas sociales, ambientales y económicos para aliviar los efectos adversos del cambio climático, actuales y futuros, así como para tomar ventaja de nuevas oportunidades. ${ }^{7}$ Desde ese momento ha habido "una explosión del interés" 8 por proponer y documentar adaptaciones a lo largo del mundo a fin de demostrar que se trata de una herramienta con alta potencialidad de éxito para afrontar los impactos del cambio climático. En el sector de la agricultura por ejemplo, la adaptación al cambio climático es una meta a la que aseguran se llegará mediante el desarrollo la agricultura climáticamente inteligente, esto es, de tecnologías que aseguren altos niveles de productividad.

La sostenida e incluso cada vez mayor atención puesta en el paradigma de la adaptación se ha producido por dos razones principales. La primera razón del interés es la asociación entre adaptación, cambio climático y desarrollo. Guiados por la definición dominante del cambio climático señalada al inicio y habiendo reconocido la magnitud de los impactos, académicos, líderes internacionales, economistas y ambientalistas, entendieron que el cambio climático podría ser una fuente de perturbación a los caminos del desarrollo. ${ }^{9}$ Así la adaptación fue emergiendo como una de las vías para evitar la inviabilidad del desarrollo y sus inversiones. De este modo se entiende que sean las principales instituciones internacionales de desarrollo, como el Programa de las Naciones Unidas para el Desarrollo (PNUD), el Banco Mundial o la misma Organización para la Cooperación y el Desarrollo Económicos (OCDE), las principales financiadoras de las intervenciones que introducen al cambio climático como un objetivo clave de la política de desarrollo. La segunda razón se vincula a la primera y se refiere al papel de la adaptación como alternativa a la que en ese momento era la vía de salida más difundida, la mitigación. Como se explicó al inicio, frente a la mitigación la adaptación apareció como una opción más amplia que podía ser desarrollada en distintas escalas. Además, era un complemento que quitaba presión a los límites de las emisiones de gases de efecto invernadero.

La adaptación introdujo también un conjunto de conceptos asociados como el de vulnerabilidad, resiliencia, gestión adaptativa y capacidad de adaptación. Todos

\footnotetext{
7 IPCC, 2001.

8 Adger, 2005, p,75.

9 Taylor, 2014; Giddens, 2009.
} 
ellos dan forma al encuadre dominante del cambio climático y contribuyen así a sostener el papel central de la adaptación en el marco de las respuestas al mismo. Tal como es formulada en el marco de la política internacional la adaptación exhibe una alta coherencia y opera como dispositivo conceptual incuestionable y global $^{10}$ así como una práctica necesaria. No obstante, es un paradigma cuyos usos no explicitan sus supuestos y asumen de manera acrítica una serie de claroscuros. El cuestionamiento más amplio y general es que propone un tipo de soluciones que no cuestionan ni buscan transformar las causas profundas que originan el fenómeno del cambio climático. Y esto porque adaptar es precisamente realizar ajustes de diferente consideración a las prácticas actuales pero no transformarlas en profundidad. No cuestiona sino que busca sostener las actividades que originan el cambio climático (especialmente los modelos de desarrollo y consumo) y de este modo perpetuar el paradigma económico que es la causa profunda de la actual crisis ambiental global y del cambio climático. La adaptación implica entonces una forma de consolidación de las diferencias de poder y recursos en la que una distribución inequitativa de los riesgos y las recompensas se incorporan en las relaciones sociedad-naturaleza de los entornos en que las personas viven.

Para comprender estas críticas es necesario explicitar los supuestos que subyacen a este paradigma en su versión dominante así como sus implicaciones más críticas.

\section{Los orígenes funcionalistas y evolutivos de la adaptación.}

La adaptación tal como la conocemos hoy es una idea con origen en la biología evolutiva. Se refiere a la relación entre una especie y su medio ambiente tanto "en el sentido débil de articulación con el medio como en el sentido fuerte de evolución para adaptarse a él." ${ }^{11}$ Posteriormente reelaborada dentro de los parámetros de la ecología cultural la idea de adaptación traslada el análisis desde la especie a la sociedad y de este modo el enfoque vincula la evolución natural con el cambio social. ${ }^{12}$

En el marco de la ecología cultural la adaptación es entendida como el proceso por el cual los sistemas sociales se autorregulan frente a las fluctuaciones y cambios en la composición y estructura de sus ambientes en el corto y largo plazo. ${ }^{13}$ Estas relaciones, guiadas fundamentalmente por la cultura, son respuestas per se a las condiciones y dinámica del entorno natural, de modo que son estrategias de adaptación. ${ }^{14}$ Así planteadas, de las estrategias de adaptación de los seres humanos se esperaba una evolución cultural más amplia que podría re-alinear las actividades humanas y sus sistemas de creencias con las exigencias de entornos externos cambiantes. Entendida desde aquí entonces la adaptación es la vía por la cual los sistemas tienden hacia al balance homeostático (o cambian a través de equilibrios

10 Taylor, 2014.

11 Harrison, 1993 en Taylor, 2014, p. 55.

12 Watts, 2015.

13 Rappaport, 1979, p.145.

14 Rappaport 1979, p. 243. 
dinámicos). Las raíces funcionalistas de esto se dejan ver no sólo en que los sistemas son capaces de autoregularse sino que los valores de las variables de los mismos tienden al equilibrio general. ${ }^{15}$

Entender la adaptación del modo que la ecología cultural lo hace, conlleva al menos dos implicaciones problemáticas. Por un lado, asumir la existencia de un orden ecológico o adaptativo trascendente al que la humanidad debería orientarse de manera lineal. ${ }^{16}$ Segundo, que el patrón de la acción colectiva puede ser entendido en un orden más amplio, sistémico y predictivo. ${ }^{17}$ En este esquema, la cultura tiene un papel sumamente relevante: es una estrategia por medio de la cual los sistemas sociales logran adaptarse a su medio. En esta conceptualización han sido fundamentales los estudios de Rappaport ${ }^{18}$ para quien los rituales son una especie de termostato del equilibrio: cuando alguna variable del sistema se sale de control, el ritual las corrige y las coloca en una situación nuevamente viable. En la explicación sobresale el carácter mecanicista de esta teoría de la adaptación: la regulación del medio ambiente ocurre a espaldas de los actores a través de estos "termostatos culturales". ${ }^{19}$

Y aquí aparece el primer aspecto problemático de este modo de entender la adaptación. Al proponer la centralidad de los sistemas de creencias arraigados para la homeostasis, el explícito funcionalismo de la adaptación funciona como marco para explicar la degradación ambiental como el resultado de prácticas sociales de gestión de la tierra, de los recursos y la naturaleza en general basadas en creencias arraigadas, pero tradicionales y anacrónicas en un mundo en rápido cambio. ${ }^{20}$ Semejante postulado resulta extremadamente problemático para aquellos enfoques que, como la ecología política, posteriormente se preocuparán por introducir la dimensión del capital en la explicación de la crisis ambiental y las transformaciones climáticas en particular. ${ }^{21}$ Es este el principal punto de contraste entre la mirada de la ecología cultural y la ecología política respecto de la adaptación. La ecología cultural atribuye las causas de la degradación ambiental, entiéndase, los problemas de la degradación de la tierra, el suelo la erosión y la deforestación a conductas de campesinos irracionales que no lograron adaptarse adecuadamente a los estímulos sociales y ambientales cambiantes.

En contraste con este localismo autorregulador de la adaptación, la ecología política sitúa los procesos dentro de una serie multiescalar de fuerzas causales. En lugar de orden natural el ecosistema planetario es entendido como un sistema ecológico en funcionamiento y en evolución en el que la naturaleza y el capital se pro-

15 Müllaer-Seichter, 2016.

16 Watts, 2015.

17 Robbins, 2009, p. 33.

18 Rappaport, 1979.

19 Watts, 2015.

20 Blaikie, 1985.

21 De hecho, como indican los críticos, la cultura como agente de transformación ambiental es interrogada a través de casos de estudios en comunidades aisladas de la modernidad y de los procesos de acumulación capitalista. Neuman, 2005. 
ducen y reproducen constantemente. Taylor ${ }^{22}$ nos recuerda que Piers Blaikie ${ }^{23}$ ya había enfatizado sobre cómo las regiones rurales revelaban los complejos resultados de las formas coloniales de gestión de la tierra y la incorporación a las relaciones capitalistas de productos básicos, las cuales tienen efectos diversos y contradictorios sobre las relaciones sociales locales y los paisajes ambientales. En la lectura de la ecología política la degradación ambiental en contextos rurales está altamente relacionada con la integración de la producción agrícola en la dinámica de acumulación regional e internacional que creaba nuevas formas de extracción de excedentes que alteraron el tejido socioecológico de las regiones rurales. ${ }^{24}$ Con ello reeemplazan la explicación de la ecología cultural que atribuye la causa a las conductas tradicionales de campesinos irracionales que no se adaptan adecuadamente a estímulos socioambientales cambiantes. Watts explica que la transformación de los ambientes agrarios y las nuevas formas de marginalidad de aquí derivadas crearon las bases sobre las cuales los campesinos fueron presionados para actuar cada vez más como agentes de degradación ambiental en una lucha por satisfacer sus necesidades de subsistencia..$^{25}$ En definitiva, a los desequilibrios de las faltas de adaptación contraponen especialmente en contextos rurales la base capitalista de la degradación ambiental. Esto significa una fuerte apuesta por una explicación diferente en la que el capital necesariamente privatiza, mercantiliza y comercializa todos los aspectos de la naturaleza.

Las raíces funcionalistas de la adaptación son determinantes para los planteos actuales de la misma en el marco del cambio climático. Por un lado, la adaptación funcionalista inspira a la nueva adaptación pero no son lo mismo. El paradigma de la adaptación del IPCC, o teoría adaptativa de segunda generación como la llama Watts, pretende salir de la simpleza de las analogías orgánicas y enfatizar que las propiedades de los sistemas complejos actuales - caracterizados por el procesamiento de información, el comportamiento colectivo complejo, la no linealidad - aseguran una adaptación y resiliencia continua. Sin embargo, a pesar de estos intentos superadores no logra despojarse de la naturalización de las relaciones sociales. Tampoco consigue evidenciar las relaciones de poder y los procesos de extracción y acumulación de plusvalía en las relaciones entre ser humano y medio ambiente. ${ }^{26}$ Es más, supone en parte un retroceso en la explicación de los cambios socioecológicos porque pasa por alto sin mayor preocupación el amplio desarrollo crítico que la ecología política y otros enfoques han realizado de este paradigma.

22 Taylor, 2014.

23 Blaikie, 1985.

24 Robbins, 2009; Van der Ploeg, 2010.

25 Watts, 1983.

26 Robins, 2004. 


\section{La adaptación reconceptualizada: de la ecología cultural al cambio climático.}

La reconceptualización de la adaptación a la luz del cambio climático se desarrolla, como ya se mencionó, junto a la idea de entender al clima como un atentado al desarrollo, una amenaza al modo de bienestar que de él se espera. Como se dijo al inicio, el clima representa un poderoso agente de anti-desarrollo que de no ser controlado haría retroceder los ya desiguales logros de la era moderna. ${ }^{27}$ Según este razonamiento, si el clima cambia en una dirección que amenaza los parámetros existentes y futuros del bienestar humano la humanidad deberá reaccionar adaptándose a través de un proceso planificado de ajuste. ${ }^{28}$ Retomando el argumento inicial, la adaptación emerge sobre esta base en la academia y la política como una de las más importantes vías de solución a ello.

El paradigma de la adaptación tendría la virtud de hacer que el cambio climático sea algo legible para la planificación gubernamental. Y si es legible es gobernable. Aquí reside para la ecología política gran parte del éxito de la adaptación: su importancia en la formulación de políticas contemporáneas depende menos de su integridad conceptual que de su capacidad para hacer manejable lo inmanejable. La adaptación se convierte en un proceso técnico de ingeniería social planificada al que se le otorga capacidad de definir qué tipo de decisiones deberían tomar los actores y los hogares para gestionar riesgos como la sequía o las inundaciones por medio de intervenciones gerenciales y tecnocráticas. Asigna responsabilidades y elige quienes serán financiados para lograr esas adaptaciones. Y por sobre todo se articula como un discurso que aporta soluciones técnicas, y por tanto, fuera de lo político. Es común que en los contextos rurales la adaptación al cambio climático se represente de manera reiterada e indiscutida, a partir de la incorporación de tecnologías: en el uso del agua, las semillas, los fertilizantes y agroquímicos, etc. La necesidad de hacer una agricultura eficiente que sea capaz de afrontar las amenazas climáticas se impone como meta y contenido indiscutible de la adaptación. “Si las sequías afectan los cultivos hay que desarrollar semillas más resistentes. En un caso extremo, si retrocede un glaciar y se pierde una fuente de agua, habrá que mover una comunidad entera. Lo importante es adaptarse a lo que hay y a lo que vendrá". ${ }^{29}$ Estas palabras con las que el responsable de Adaptación al Cambio Climático de Argentina explica la posición de su institución es una muestra de estas pretendidas soluciones técnicas. La adaptación refuerza condiciones previas de inequidad porque toda la maquinaria se orienta a generar adaptaciones a un modelo que ya es en sí mismo inequitativo.

Pero la adaptación no sólo es técnica sino que es inherentemente política: las propuestas contienen una serie de supuestos básicos que condicionan la forma en que concebimos los vínculos entre el cambio social y el ambiental. Al hacerlo, el lenguaje de la adaptación legitima formas particulares de conocimiento, gobernan-

27 Giddens, 2009.

28 IPCC, 2014.

29 Pagura, 2018. 
za y política, pero lo hace de manera silenciosa sin explicitar los supuestos analíticos y normativos que están incorporados en sus fundamentos conceptuales. ${ }^{30}$ La adaptación es un proceso político además, porque los ajustes que propone a los medios de subsistencia de los actores tienen resultados desiguales. Individuos y grupos interactúan y compiten para promover sus propios intereses discretos. ${ }^{31}$ De este modo, las propuestas que de ella emanan son débiles porque dedican escasa o nula atención a las raíces históricas de las vulnerabilidades estructurales que determinan las configuraciones de los actores en el territorio. Así, primero la literatura sobre adaptación, y luego las políticas, sistemáticamente eluden las cuestiones fundamentales relativas al control históricamente configurado y jerarquizado sobre la tierra, el agua, el capital y el trabajo que suelen caracterizar a las regiones rurales (pero también a las ciudades) y que distribuyen de manera desigual los riesgos y las recompensas dentro de ellas. Un pequeño productor de una zona árida de los Andes Centrales en Argentina puede haber seguido minuciosamente las recomendaciones de adaptación a la disminución de caudal del río construyendo un pozo y optimizando su sistema de riego. Pero las escasas rentabilidades asociadas a precios bajos fijados por mercados globales que operan en ausencia de políticas locales de protección de la agricultura regional, neutralizan cualquier resultado positivo de estas adaptaciones típicamente propuestas. ${ }^{32} \mathrm{Al}$ desvincular estas prácticas y sus expresiones locales de vulnerabilidad de las estructuras de poder y privilegio más amplias, la adaptación opera como un concepto despolitizador porque reduce las complejas relaciones socioecológicas a un llamado abstracto para defender a las comunidades de perturbaciones y amenazas ambientales externas. Continuando con el ejemplo anterior, se asume como natural, puesto que viven en un desierto, que los campesinos de las áreas no irrigadas de las cuencas andinas deban desarrollar capacidades creativas para adaptarse a la escasez. Sin embargo, las adaptaciones de ellos esperadas naturalizan también el hecho de que los magros caudales que hoy reciben son consecuencia de usos cuenca arriba efectuados por actores que llegaron después que ellos pero lo hicieron con el poder de capturar esos caudales. La mirada de la adaptación sobre el cambio climático lo ve como si este fuera un elemento más que configura las dinámicas de los contextos rurales. Sin tener en cuenta que éste no actúa de manera aislada sino que se combina necesaria e indisolublemente con otros procesos no biofísicos, como los modos de comercialización de la agricultura, las relaciones de propiedad, las formas de acumulación de capital. ${ }^{33}$ No se trata de negar la importancia del clima sino de verlo a la luz de estas transformaciones de los contextos rurales. El análisis de Watts en su clásica obra Silent Violence es esclarecedor para demostrar cómo las formas tradicionales de adaptación a la sequía de los agricultores y pastores del Sahel, en África, se ven afectados por las redes globales del capitalismo contemporáneo. El análisis muestra de qué manera, a pesar de que

30 Taylor, 2014, p. 52.

31 Eriksen y Lind, 2009, p. 818.

32 Montaña y Mussetta, 2016.

33 Dietz, 2013; Montaña, 2008; Van der Ploeg, 2010. 
la sequía es algo natural, la hambruna derivada de ella es un factor producido por las relaciones de economía política que han alterado las formas tradicionales de adaptación a esa falta de agua. ${ }^{34}$

\section{Los intentos de elaborar una adaptación más crítica.}

No sería preciso no reconocer en este punto la abundante bibliografía con la que muchos autores preocupados por las limitaciones recientemente señaladas de este paradigma tratan de superarlas, especialmente incorporando las nociones del contexto a la explicación de la adaptación. Uno de los intentos por mejorar este enfoque es el que partiendo de la hipótesis que la adaptación de unos puede implicar mayor vulnerabilidad de otros, desarrolla el concepto de "maladaptación" para abordar los efectos o consecuencias no deseadas que podrían aumentar la vulnerabilidad de ciertos grupos, ya sea en el mismo lugar o en otro. Estas ideas generalmente también incorporan el concepto de vulnerabilidad, para sostener que esta última puede ser transferida, de unos grupos a otros cuando proponemos acciones de adaptación. Entre estos estudios, se destaca el trabajo de Eakin ${ }^{35}$ que ofrece ejemplos del sector cafetero; el de Klein ${ }^{36}$ que utiliza un ejemplo de medidas de adaptación costera y el de Atteridge y Remling ${ }^{37}$ quienes rastrean las dimensiones sociales y económicas de los impactos de las adaptaciones del sector cafetero en Colombia. Además, entre estos trabajos se destaca el trabajo de Adger et al., quienes a través del concepto de "efectos espaciales indirectos" ( spatial spillovers) demuestran cómo una adaptación típica para zonas inundables, como lo es la construcción de nuevos terraplenes de inundación, protegen a una comunidad mientras a la vez aumentan la vulnerabilidad de las comunidades cuenca abajo. ${ }^{38}$ La lógica de los "ciclos o bucles causales" (causal loops) también fue utilizada para estudiar la asociatividad de las múltiples causas de la vulnerabilidad producto de las adaptaciones al cambio climático. ${ }^{39}$ Otro conjunto de observaciones clave que realizan estos trabajos es que las medidas adoptadas para adaptarse al cambio climático pueden tener en sí mismas importantes implicaciones para la justicia porque sus beneficios y costos se distribuyen con frecuencia de manera que consolidan o exacerban las vulnerabilidades actuales en lugar de reducirlas. Es decir, las estrategias y medidas de adaptación crean sus propios ganadores y perdedores en función de la elección de soluciones para la gobernanza de las respuestas colectivas e individuales a los riesgos climáticos. ${ }^{40}$

Por su parte, también las producciones del IPCC más recientes reconocen la importancia de las fuerzas y condiciones no climáticas que condicionan cualquier proceso de adaptación. Sin embargo, estas dinámicas se reducen típicamente, en

34 Watts, 1983.

35 Eakin et al., 2009.

36 Klein et al, 2007.

37 Atteridge y Remling, 2013.

38 Adger et al, 2005.

39 Navarrate, Gómez y Gallopín, 2007.

40 Paavola, Adger y Huq, 2006. 
palabras de Bassett y Fogelman ${ }^{41}$, a factores próximos cuyas raíces causales nunca se teorizan ni pretenden ser modificadas. Como tal, la historia de cómo se ha llegado a las actuales condiciones, con el desigual poder sobre el uso y apropiación de la naturaleza, se naturaliza junto con la excepcionalidad del cambio climático. Sobre esta base, como dice Elizabeth Shove, "la política de la adaptación procede sobre la base de un relato extremadamente simplificado del mundo social". ${ }^{42}$

Los estudios que se esfuerzan por ampliar las bases explicativas de la adaptación son valiosos y deben ser reconocidos. Pero todos ellos proporcionan instrumentos para mejorar las acciones de adaptación, no para cambiar el enfoque. Es decir, son estudios que permanecen dentro de los paradigmas de la adaptación y no cambian sus supuestos. En línea con la ecología política, estos intentos de hacer de la adaptación un paradigma más amplio, son limitados porque son elaborados bajo los mismos problemáticos supuestos que aquí estamos tratando de desentrañar. Sus fundamentos siguen presentes y son los que hacen que sea un paradigma restrictivo a pesar de las sucesivas reformulaciones.

\section{Naturaleza, clima y sociedad}

Como se dijo ya, la definición más difundida de adaptación al cambio climático, la provista desde el IPPC, es la de "un proceso de ajuste". En esta definición, clima y sociedad están representados como dos sistemas distintos (uno biofísico natural y otro cultural social) que se relacionan mutuamente. La sociedad afecta y degrada el medio ambiente: a través de la quema de combustibles fósiles, la emisión de gases de efecto invernadero, la devastación de especies animales y vegetales, la extracción indiscriminada de minerales. Todo ello incide en los procesos atmosféricos resultando en lo que los climatólogos llaman cambios externos del clima o cambio climático antropogénico. ${ }^{43}$ El clima a su vez, repercute en los procesos sociales a través de una serie de estímulos y tensiones que van desde eventos climáticos extremos hasta cambios más sutiles, pero no por ello poco contundentes, en la temperatura y las precipitaciones. De manera similar a lo que señalábamos sobre la ecología cultural, aquí también la adaptación emerge como el proceso de ajuste, de reconciliación entre dos sistemas relacionados externamente que se han desajustado, desincronizado. Los postulados de la herencia funcionalista de la adaptación original permanecen vigentes.

La relación entre clima y sociedad es conceptualizada como una relación entre entidades diferentes. A los procesos naturales y a los sociales se les atribuyen dinámicas distintas. De este modo, la adaptación actualiza una discusión -tan antigua y estudiada como controversial e inconclusa- sobre la relación sociedad y naturaleza. Es decir, la manera en que la adaptación entiende la relación entre clima y sociedad, se inscribe en una relación más general, previa y de larga data, entre naturaleza y

41 Bassett y Fogelman, 2013.

42 Shove, 2010, p. 1277.

43 El clima también sufre procesos de cambios internos, propios de la evolución de ese sistema. 
sociedad. De hecho, esta separación rígida entre clima y sociedad no es propia de los debates en torno al cambio climático o la crisis ambiental reciente sino que es producto de la distinción ontológica entre el mundo social y el mundo natural típica del pensamiento occidental y modernista. ${ }^{44}$ La conceptualización dicotómica entre clima y sociedad conduce inexorablemente a la representación del cambio climático como una fuerza exógena, una amenaza generada externamente y no como el producto de las constantes transformaciones de las relaciones sociales. La adaptación es un paradigma diseñado para resaltar lo primero y soslayar lo segundo: tiende a detener los procesos socio-ecológicos de producción, a aislar y extraer al cambio climático de esta instantánea como un agente causal que posee su propia dinámica. Una vez convenientemente despojado de tal contexto el clima se vuelve a insertar como una serie de perturbaciones externas. ${ }^{45}$

La adaptación, al limitarse a evaluar las mutuas influencias entre naturaleza/ clima y sociedad como dos entidades distintas asume que el medio ambiente es un contexto preconcebido sobre el que la sociedad actúa. El contrapunto principal que la ecología política elabora ante esta visión postula en cambio que la naturaleza es socialmente producida y construida. Esto implica un punto de partida diferente; implica preguntarse por cómo se producen activamente los entornos socioecológicos, cómo la naturaleza es producida materialmente por medio de prácticas sociales, tanto en su dimensión social como climática. El supuesto es diferente y entiende que la sociedad es un agente de transformación ambiental en los procesos de reproducción activa de sus vidas y sus entornos. La naturaleza no es la superficie de materialidad sobre la que se inscribe la historia humana sino que la historia es el proceso en el que tanto las personas como sus entornos son continuamente hechos realidad unos a otros. ${ }^{46}$ La producción social de la naturaleza se contrapone a la dicotomía naturaleza-cultura/sociedad: politiza las explicaciones clásicas sobre degradación ambiental y descarta la idea de que los modos de producción operan al margen de la naturaleza. ${ }^{47}$ La dinámica de transformación de los ambientes rurales y urbanos es constante y se encuentra marcada por los fluctuantes ritmos de acumulación de capital, el cambio tecnológico, las relaciones comerciales, las prácticas políticas. El cambio climático no es una excepción ni una externalidad a estos procesos. No es una variable independiente sino que es otro elemento persistente más - que es tanto causa y efecto- de la producción activa de seres humanos y sus entornos de vida obstinadamente desiguales.

La reproducción de esta ontología dualista que asume la adaptación, resulta problemática además porque es una simplificación que no logra describir las complejidades de la crisis ambiental que vivimos. Como señala Jason Moore, una vez que indagamos en profundidad los procesos a través de los cuales se producen las sociedades y sus entornos "la visión cartesiana de una dicotomía inherente entre la

44 Castree, 2005.

45 Taylor, 2014.

46 Ingold, 2000, p. 87.

47 Bustos et al, 2015. 
naturaleza y la sociedad se vuelve teóricamente arbitraria y empíricamente engañosa: si no, trate usted de trazar una línea divisoria en lo social y lo natural en el cultivo y consumo de alimentos, en un arrozal o un campo de trigo; ¿dónde termina el proceso natural y comienza la vida social?". ${ }^{48}$ En el mismo sentido, Svampa remarca que la persistencia de la dicotomía es un obstáculo para superar la crisis ambiental actual en tanto la posibilidad de abrir nuevos caminos exige un enfoque post dualista y relacional. ${ }^{49}$

Entonces, ¿por qué el discurso de la adaptación se basa en y reproduce un marco conceptual que ordena las relaciones socio-ecológicas de esta manera dicotómica? La respuesta de la ecología política es clara y contundente. La dicotomía es útil porque evita discutir el papel del capitalismo en la crisis ambiental en general y el cambio climático en particular así como las consecuencias desiguales de esa crisis sobre distintos grupos de actores. Al abstraerse de las formas en que los seres humanos coproducen sus entornos de vida en todas las escalas, los conceptos de vulnerabilidad, resiliencia y capacidad de adaptación se presentan abrumadoramente como temas de "aquí y ahora" en los que las cuestiones de la adaptación están radicalmente separadas de las trayectorias de cambios socioecológicos más largas y cuyas fuerzas causales se extienden más allá de los lugares en los que se manifiestan. ${ }^{50}$

Son muchos los ejemplos que demuestran que los eventos climáticos y meteorológicos están intrínsecamente entrelazados con las prácticas del monocultivo, de la deforestación y desmonte de la extracción de minerales, de impermeabilización de humedales. Estas prácticas de transformación socio-ecológica impactan en dimensiones sociales y climáticas. Sin una mirada histórica que entienda el peso que ha tenido el capitalismo en las transformaciones climáticas y especialmente atienda los extremos niveles de desigualdad entre unos grupos y otros el análisis continúa siendo estrecho y limitado para encontrar salidas a los patrones de desigualdad social y las perturbaciones climáticas. La adaptación evita exitosamente considerar estas cuestiones.

Aún la idea del antropoceno, que se elabora en torno a la centralidad de las causas humanas de la crisis e intenta desafiar la dicotomía sigue centrándose en los impactos de la crisis sobre la humanidad, ${ }^{51}$ sigue planteando el problema en términos globales sin poder incluir las diferencias sociales y considera al ser humano como especie sin abordar las complejidades ni las diferencias sociales. ${ }^{52}$ Tampoco brinda soporte teórico o analítico para entender las transformaciones socioecológicas como inherentemente desiguales e innatas a la dinámica del capitalismo. ${ }^{53}$

48 Moore, 2013.

49 Svampa, 2018.

50 Swyngedouw, 2011.

51 Patel y Moore, 2018.

52 Palacio, Vargas y Hennessy, 2017.

53 La idea de capitaloceno pretende aportar los elementos críticos de los que adolesce el antropoceno. Para una explicación de este concepto ver: Patel y Moore 2018. 


\section{Adaptación, resiliencia y gestión del riesgo}

El último argumento con el que reconstruimos la crítica a la adaptación destaca cómo este paradigma, junto a la idea de resiliencia, asume una forma de gestión del riesgo que se inscribe en un tipo de racionalidad calculativa que es propia de las formas de los gobiernos liberales avanzados. ${ }^{54} \mathrm{La}$ adaptación presenta la idea de que el futuro incierto (y en gran medida catastrófico) que el cambio climático augura es fatible de ser pensado, calculado, administrado. De este modo transmite el mensaje de que es posible prepararse para los impactos del cambio climático y en alguna medida remediarlos. La adaptación desafía así las incertidumbres y perturbaciones que el cambio climático introduce.

Por otra parte la resiliencia, concepto originalmente desarrollado dentro de la ecología y reconfigurada luego desde las ciencias sociales y socio-ecológicas, comparte con el pensamiento adaptativo la búsqueda de una relación entre la durabilidad y la flexibilidad de los sistemas. Independientemente de su objeto de referencia específico (sequía, eventos extremos, inundaciones, finanzas) la adaptación y la resiliencia se rigen por una cultura de la precaución que busca anticiparse a los riesgos y en última instancia aminorar o evitar impactos futuros. ${ }^{55}$ Entonces, la calculabilidad de los riesgos que aleja la incertidumbre trae aparejada la seguridad. Se instalan así los relatos de las seguridades, hídrica, alimentaria, jurídica, climática.

Por otro lado, esta lógica que busca hacer que los riesgos sean calculables, se inscribe en una racionalidad calculativa en la que la incertidumbre del futuro es incorporada en decisiones y prácticas en el presente, aquí y ahora. ${ }^{56}$ Un rasgo de esta racionalidad, es que la gestión del riesgo tiende privatizarse. Esto es, a la adaptación le subyace una tendencia a la privatización de la gestión del riesgo que consiste en que son los actores individuales quienes tienen la responsabilidad de desarrollar prácticas de adaptación y ajuste al cambio climático. Las políticas de adaptación y resiliencia son planificadas para una pluralidad de agregaciones que vistos a gran escala son poblaciones en riesgo frente al cambio climático, pero en pequeñas escalas son agregados de productores, de consumidores, de usuarios individuales de bienes naturales aparentemente iguales, ${ }^{57}$ que se ven compelidos a desarrollar prácticas autónomas y casi siempre individuales para ajustarse a las nuevas condiciones. Este es un proceso que se da en paralelo a la retracción de las técnicas socializadas de administración del riesgo propias del Estado de bienestar, dadoras de derechos sociales.

La adaptación y la resiliencia invocan así un nuevo sentido de homo economicus, o un homo prudens en palabras de Dean,$^{58}$ un tomador de decisiones en sistemas auto-organizados y adaptables que se enfrentan a amenazas catastróficas y se con-

54 Foucault, 2007.

55 Amoore, 2013.

56 Rose, 1996.

57 Watts, 2015.

58 Dean, 1998. 
vierten en prudentes empresarios de sí mismos. ${ }^{59}$ Así, la lógica de la racionalidad calculativa que subyace a la adaptación y la resiliencia, que implica un proceso de individualización del riesgo junto a la cultura de la prevención, es analizada por algunos autores como una manera de gobernar sin gobernar. Esto es, de gobernar no ya a través de un complejo aparato estatal sino a través de acciones prudentes y responsables de sujetos individuales en nombre de ellos mismos, ${ }^{60}$ una especie de gestores de fondos de cobertura para una vida contingente, turbulenta e impredecible. ${ }^{61}$

De este modo, emerge aquí otro aspecto político que subyace a la adaptación: la adaptación es política también porque implica un tipo particular de gestión de las vidas individuales. La adaptación se inscribe en una reconfiguración del poder político que no es simplemente entendida desde la dicotomía estado/mercado. Los nuevos mecanismos de gobierno son diseñados y programados por autoridades políticas para unir los cálculos y las acciones de un amplio conjunto de individuos heterogéneos con objetivos políticos, y gobernarlos a la distancia a través de la instrumentalización de la autonomía regulada. ${ }^{62}$ Este análisis nos lleva al concepto de biopolítica como la administración y regulación de los procesos de vida. ${ }^{63}$

A la luz de estas interpretaciones, la búsqueda de resiliencia y de adaptación para los autores de la ecología política, no puede separarse de la búsqueda de sistemas de poder estabilizados. Lejos de ser una propiedad sistémica, la resiliencia y adaptación implican una posible institucionalización de las diferencias de poder en el que una distribución desigual de los riesgos y las recompensas son parte constitutiva de los entornos vividos. ${ }^{64}$ El pensamiento resiliente o adaptativo es una de las formas contemporáneas de liberalismo, un medio por el cual todos somos supuestamente capaces de anticiparnos y tolerar los disturbios, peligros y contingencias radicales de habitar un mundo complejo y amenazado por el cambio climático. No caben aquí explicaciones a cómo y por qué la resiliencia de unos puede ser la subyugación de otras ni por qué algunas formas de auto-organización, la de los mercados, triunfa sobre las demás.

\section{Reflexiones finales: menos adaptación y más crítica al modelo actual de producción de naturaleza}

La adaptación es un enfoque con un alto protagonismo entre las opciones que las agencias de financiamiento, los organismos de gobierno y los centros de investigación proponen como solución para hacer frente el cambio climático. La corriente principal de la ciencia y la política del cambio climático la presenta como una respuesta evidente y natural ante los impactos y amenazas futuras del cambio climá-

59 Foucault 2007, p. 241.

60 Dean, 1998.

61 Watts, 2015.

62 Rose, 2006, p.157.

63 Foucault, 2007.

64 Cannon y Mueller-Mahn, 2010 en Lampis 2012, p. 38. 
tico, como una necesidad impostergable e incuestionable. A pesar de la aparente evidencia y naturalidad de la adaptación el argumento de este artículo sostiene la necesidad de tomar con extrema cautela tales formulaciones.

Para sostener esta idea y con apoyo en el análisis de la ecología política se han explicitado aquí sus supuestos e implicaciones, muchos de los cuales condicionan los alcances de las respuestas concretas que de ella puedan derivarse. De manera general, se dijo que la adaptación propone ajustes moderados que no son suficientes para afrontar el problema que el cambio climático y la degradación ambiental nos imponen. Pero además, despliega un conjunto de herramientas que refuerzan las desigualdades. En primer lugar, se señaló el carácter mecanicista y biologicista de la adaptación en parte heredado desde sus orígenes funcionalistas. Junto a ello, su adhesión a una ontología dualista de la relación sociedad-naturaleza/clima. Desde esta dicotomía, que fundamenta y da coherencia el concepto de adaptación ${ }^{65}$ ella emerge como un proceso de ajustes técnicos planificados que, al mediar las amenazas climáticas actuales y proyectadas, podría devolver al sistema social y al natural una coexistencia más equilibrada, más armónica. En tercer lugar, se explicó cómo en el enfoque dominante la adaptación se complementa con el concepto de resiliencia. Así resaltando las supuestas propiedades auto-organizadoras de los sistemas sociales y la gestión privada de los riesgos, constituyen una métrica calculadora que es funcional al neoliberalismo.

Todos estos supuestos en los que se funda la corriente dominante de la adaptación remueven completamente del análisis los componentes políticos del cambio climático y de las soluciones propuestas. Asimismo evitan discutir la construcción social de la naturaleza característica central para el estudio de la crisis climática y ecológica. Estas limitaciones no son reconocidas por los centros de gobernanza climática. Los postulados no se explicitan y son naturalizados en las narrativas dominantes.

Una vez repasadas las críticas y llegados a este punto es necesario formular la pregunta acerca de si es posible redefinir el enfoque de la adaptación para hacer emerger desde allí respuestas a la crisis climática que superen los problemas de la versión dominante. Los interrogantes entonces son: ¿es posible reformular la adaptación para convertirla en un concepto útil para resolver los problemas de desigualdad que el cambio climático evidencia?; ¿es posible adscribir la adaptación a una teoría crítica de las relaciones sociedad-naturaleza, como la que plantea la ecología política?; ¿es posible dotar a la adaptación del contenido político del cual sus postulados se encargan de mantenerla alejada?; ¿ es posible rescatar algunas potencialidades del enfoque para pensar desde allí respuestas y soluciones al cambio climático? Los argumentos desarrollados en este artículo llevan a sostener que la adaptación no es la salida. El intento por una reformulación crítica del enfoque no es una tarea que lleve a una apropiada manera de comenzar a pensar horizontes alternativos a

65 Watts, 1983. 
la crisis.

Como ya se señaló, un gran número de estudios ${ }^{66}$ han realizado intentos en este sentido, ampliando el enfoque de la adaptación sacándolo el esquema mecánico y biologicista e incorporando elementos del contexto en la explicación de las diferencias en los impactos. Sin duda estos trabajos son superadores del paradigma de la adaptación propiamente dicho porque condicionan el potencial de la adaptación a explicaciones estructurales del contexto de los actores implicados y se esfuerzan por analizar los drivers de las vulnerabilidades antes que los impactos en sí mismos. Así dejan entrever la dimensión política del problema porque enfatizan los aspectos de la desigualdad en las condiciones de origen. No obstante, estos estudios no son suficientes porque rodean el problema de la construcción social de la crisis climática y ecológica, pero no lo abordan. Aunque asumen como centrales las desigualdades sociales y la necesidad de asociar las salidas a la crisis climática a la pobreza (aun los que hablan de justicia y equidad climática) permanecen en la lógica de atender los impactos del cambio climático y no necesariamente introducen al análisis las causas de la crisis climática. A estos estudios debemos reconocerles su potencial para estudiar las causas de la vulnerabilidad a los impactos, es decir, las causas de las consecuencias desiguales de la crisis climática sobre los distintos grupos sociales, pero no las causas de la crisis socioecológica.

Para pensar respuestas a la crisis climática será necesario acudir a enfoques que habiliten un diagnóstico crítico de las bases del desarrollo capitalista, del perfil metabólico de nuestras sociedades, del modelo de producción de alimentos y de consumo y por qué no, del modelo de producción y circulación de conocimientos. La adaptación es un paradigma que no admite lugar a estos temas. Propuestas genuinas a que lleguen a las causas de las crisis climática y ecológica sólo podrán derivarse de diagnósticos que reconozcan que el cambio climático es producto de algo mucho más amplio. Tal diagnóstico es imposible que pueda derivarse desde dentro del enfoque de la adaptación. Más bien, como expresa Parr, “la adaptación abona la esencia misma del capitalismo; el mundo se adapta sin eliminar la amenaza que él constituye sino por ejemplo, comprando favores como en los mercados voluntarios de carbono." 67 O peor aún, como en el caso de la agricultura, en donde la adaptación promueve una agricultura más eficiente, con mayor productividad. Y esa misma productividad implica una mayor sobreexplotación de los suelos, el agua, los bosques nativos.

Abandonar el marco de la adaptación para pensar las salidas al cambio climático implica despojarse de la idea de una naturaleza dada y objetiva y colocar en el centro de la escena las formas en que la naturaleza es producida por la sociedad. No se trata de adaptarse a un entorno natural externo cambiante. Se trata de reconocer la brutalmente desigual sobreproducción de la naturaleza. Urge reemplazar las adaptaciones por prácticas que constituyan verdaderas fisuras a este modelo.

66 Adger et al, 2005; Eakin et al, 2009; Eriksen y Lind, 2009.

67 Parr, 2013, p.146. 
Es necesario proyectar cómo "ralentizarnos rápidamente" ${ }^{68}$ y reordenar las fuerzas productivas existentes hacia fines más equitativos.

Menos adaptación y más crítica al modelo civilizatorio quiere decir revisar errores, limitaciones y problemas existentes en las principales corrientes de abordaje de la crisis climática y sus vías de salida; romper las fronteras de las disciplinas para fisurar la visión científica del cambio climático. Para ello "es crucial que los ecólogos se animen a hablar de economía y los economistas de ecología y temas sociales" sostiene Martinez Alier. ${ }^{69}$ En segundo lugar, resaltar y adoptar los enfoques que parten del supuesto de que crisis climática, crisis ecológica y modelo económico, están causalmente conectadas y dan forma a la crisis del modelo civilizatorio hegemónico, ${ }^{70}$ como algunos autores han llamado.

Finalmente, es importante rescatar aquellos trabajos que haciendo frente al pensamiento único asumen la diversidad y proponen alternativas de cambio que emanan desde otros posicionamientos epistemológicos (como los movimientos sociales) que se gestan sobre la base de la diferencia y la diversidad socio-cultural. ${ }^{71}$ El camino recorrido en este sentido es largo. Y aunque no ha logrado romper aun el círculo hegemónico de la ecogubernamentalidad (en el que la ciencia y política del cambio climático se retroalimentan en la mirada unívoca del problema que evade estas cuestiones fundamentales) son los pilares desde donde producir el conocimiento y las prácticas para el cambio.

\section{Bibliografía}

ADGER, W., ARNELL, N. y TOMPKINS, E. Successful adaptation to climate change across scales, Global Environmental Change, 2005, 15(2), p. 77-86.

AMOORE, L. The Politics of Possibility: Risk and Security Beyond Probability. Durham: Duke University Press, 2013, 232 p.

ATTERIDGE, A. y REMLING, E. The Indirect Effects of Adaptation: Pathways for Vulnerability Redistribution in the Colombian Coffee Sector. Stockholm Environment Institute, Working Paper, 2013, 10, p. 1- 40.

BASSETT, Thomas y Charles Fogelman. Déjà vu or Something New? The Adaptation Concept in the Climate Change Literature, Geoforum 48, 2013, p: 42-53.

BLAIKIE, Piers. The Political Economy of Soil Erosion in Developing Countries. Harlow: Longman, 1985, $188 \mathrm{p}$.

BUSTOS, Beatriz, Manuel Prieto y Jonathan Barton. Ecología politica en Chile: naturaleza, propiedad, conocimiento y poder. Santiago de Chile: Editorial Universitaria, 2015, 275 p.

68 Watts, 2015.

69 Martinez Alier, 2015.

70 Lowy, 2016; Svampa, 2018.

71 Algunos otros encuadres similares que comparten las bases y los puntos de llegada con estas notas la ética ecológica (Riechmann 2004), la justicia ambiental (Schlosberg 2007), el decrecimiento (Sempere 2009). 
CANNON, Terry y Detlef Müller-Mahn. Vulnerability, resilience and development discourses in context of climate change, Natural Hazards: Journal of the International Society for the Prevention and Mitigation of Natural Hazards, 2010 , vol. 55: 3, p. 621-635.

CASTREE, Noel. Nature. Nueva York: Routledge, 2005, 281 p.

DEAN, Mitchell. Risk, calculable and incalculable. Risk and Sociocultural Theory

New Directions and Perspectives, Deborah Lupton ed., New South Wales: Cambridge University Press, p. 131-159

DIETZ, Kristina. Hacia una teoría crítica de vulnerabilidad y adaptación: aportes para una reconceptualización desde la ecología política. Culturas, conocimientos, politicas y ciudadanías en torno al cambio climático, Astrid Ulloa y Andrea Ivette Prieto eds. Bogotá: Biblioteca Abierta Colección General, serie Perspectivas Ambientales, 2013, p. 19-46.

EAKIN, H., WINKELS, A. y SENDZIMIR, J. Nested vulnerability: exploring crossscale linkages and vulnerability teleconnections in Mexican and Vietnamese coffee systems. Environmental Science \& Policy, 2009, 12(4), p. 398-412.

ERIKSEN, Siri, y Jeremy Lind. Adaptation as a Political Process: Adjusting to Drought and Conflict in Kenya's Drylands. Environmental Management 43, n. ${ }^{\circ}$, 2009, p. 817-35.

FOUCAULT, M. El nacimiento de la biopolítica. Buenos Aires: Fondo de Cultura Económica, 2007, 401 p.

GIDDENS, Anthony. La política del Cambio Climático. Madrid: Alianza Editorial, 304 p.

HOWE, Joshua P. Behind the Curve: Science and the Politics of Global Warming. Seattle: University of Washington Press, 2014, 293 p.

INGOLD, Tim. The Perception of the Environment: Essays on Livelihood, Dwelling and Skill. Londres: Routledge, 2000, 465 p.

IPCC. Technical summary. Climate Change 2014: Impacts, Adaptation, and Vulnerability. Part A: Global and Sectoral Aspects. Contribution of Working Group II to the Fifth Assessment Report of the Intergovernmental Panel on Climate Change Field, C.B., et al eds. Nueva York: Cambridge University Press, 2014, p. 35-94.

IPCC. Climate change 2001: synthesis report. A contribution of Working Groups I, II and III to the Third Assessment Report of the Intergovernmental Panel on Climate change, Nueva York, 2001,235 p.

KLEIN, Richard, et al . Portfolio Screening to Support the Mainstreaming of Adaptation to Climate Change into Development Assistance, Climatic Change 84, n. ${ }^{\circ} 1,2007$, p. 23-44.

LAMPIS, Andrea. La adaptación al cambio climático: el reto de las dobles agendas. Cambio Climático, Movimientos Sociales y Políticas Públicas. Una Vinculación Necesaria. Postigo, Julio C, et al. Comps, Santiago: CLACSO, 2012, p. 29-50. 
MARTÍNEZ ALIER, Joan. Hacen falta ecólogos que se atrevan a hablar de los temas sociales. CLACSO TV, 2015, isponible en: https://www.youtube.com/ watch? $\mathrm{v}=\mathrm{mwLw}-\mathrm{KRU} \_$Yc\&t $=442 \mathrm{~s}$

MICHAELS, Patrick y Robert C. Balling. Climate of Extremes: Global Warming Science They Don't Want You to Know. Washington, D.C: Cato Institute Press, 2009, 250 p. MONTAÑA, Elma. Las disputas territoriales de una sociedad hídrica. Conflictos en torno al agua en Mendoza, Argentina. Revista Interamericana de Economía Ecológica, 2008 Vol. 9, p. 1 - 17.

MONTAÑA, Elma y Paula Mussetta. Vulnerabilidad al cambio ambiental global en

los Andes Centrales: Enunciados para un enfoque hacia la justicia climática, LASA Forum, Volume xlvii : issue 4, 2016, p. 17-20

MOORE, Jason. El auge de la ecología-mundo capitalista (I): las fronteras mercantiles en el auge y decadencia de la apropiación maxima. Filosofía, política y economía en el Laberinto, 2013, No. 38, 2013, p. 9-26.

NAVARRETE, Manuel, José Gómez y Gilberto Gallopín. Syndromes of sustainability of development for assessing the vulnerability of coupled humanenvironmental systems. The case of hydrometeorological disasters in Central America and the Caribbean . Global Environmental Change, 2007, 17, p. 207217.

NEUMANN, Roderick. Making Political Ecology. Londres: Hodder Arnold, 2005. 213 p.

PAAVOLA, Jouni, W. Neil Adger, and Saleemul Huq. Multifaceted Justice in Adaptation to Climate Change, Fairness in Adaptation to Climate Change W. Neil Adger et al eds. Cambrigde: Massachusetts Institute of Technology, 2006, p. 263-278.

PAGURA, Carlos. Las claves del plan que prepara Argentina para adaptarse al cambio climático. Ambito Financiero, 2018, disponible en: https://www. ambito.com/las-claves-del-plan-que-prepara-argentina-adaptarse-al-cambioclimatico-n4024102.

PALACIO, Germán, Alberto Vargas y Elizabeth Hennessy. Antropoceno o Capitaloceno en fricción. Des-Encuentros entre Geociencias e Historia, Ecología política latinoamericana: pensamiento crítico, diferencia latinoamericana y rearticulación epistémica, Hector Alimonda, Catalina Toro y Facundo Martín eds. Buenos Aires: CICCUS, 2017, p.265-288.

PARR, Adrian. The Wrath of Capital: Neoliberalism and Climate Change Politics. Nueva York: Columbia University Press, 2014, 232 p.

PATEL, Raj y Jason Moore. A History of the World in Seven Cheap Things: A Guide to Capitalism, Nature, and the Future of the Planet. California: University of California Press. 2017, 336 p.

RAPPAPORT, Roy. Ecology, meaning, and religion. California: North Atlantic Books. 1979, $272 \mathrm{p}$.

RIECHMANN, Jorge. Ética ecológica : propuestas para una reorientación, Montevideo: Nordan-Comunidad, 2004, 247 pp. 
ROSE, Nikolas. Governing Advanced Liberal Democracies, The Anthropology of the State. A Reader, Aradhana Sharma y Akhil Gupta, coord. Padstow Cornwall: Blackwell, 2006, p.144-162.

ROSE, Nikolas. The Death of the Social? Re-figuring the territory of government. Economy and Society. Vol. 25 Número 3, 1996 p.327-356.

SCHLOSBERG, David. Defining Environmental Justice: Theories, Movements, and Nature, Oxford: Oxford University Press, 2007, 252 pp.

SEMPERE, Joaquín. Mejor con menos: necesidades, explosión consumista y crisis ecológica, Barcelona: Crítica, 2009, 264 pp.

SHOVE, Elizabeth. Beyond the ABC: Climate Change Policy and Theories of Social Change, Environment and Planning A: Economy and Space, 2010 Volume: 42: 6, p. 1273-1285.

SMITH, Joel, Richard Klein y Saleemul Huq. Climate Change, Adaptive Capacity and Development. Londres: Imperial College Press, 2003, 298 p.

SVAMPA, Maristella. Narrativas de la crisis socioecológica en el Antropoceno, Revista Nueva Sociedad, 2018, No 278, p. 151-164.

SWYNGEDOUW, Erik. ¡La naturaleza no existe! La sostenibilidad como síntoma de una planificación despolitizada, Urban , 201 1, 26, p.41-66.

TAYLOR, Marcus. The political ecology of climate change adaptation: livelihoods, agrarian change and the conflicts of development, Londres: Routledge, 2015, 26 p.

ULLOA, Astrid, Justicia climática y mujeres indígenas en América Latina, LASA Forum, Volume xlvii : issue 4, 2016, p. 12-16.

VAN DER PLOEG, Jan Douwe. Nuevos campesinos: campesinos e imperios alimentarios. Barcelona: Icaria, 2010,430 p.

WATTS, Michael. The Political Economy of Climatic Hazards : A Village Perspective on Drought and Peasant Economy in a Semi-Arid Région of West Africa. Cahiers d'études africaines, 1983, vol. 23, n89-90, p. 37-72.

WATTS, Michael. Silent Violence. Food, famine and peasentry in Northern Nigeria. 1983, p. 660.

(c) Copyright: Paula Mussetta, 2020

(c) Copyright: Scripta Nova, 2020.

Ficha bibliográfica:

MUSSETA Paula. La adaptación como respuesta al cambio climático. Nota acerca de las contracaras de un paradigma dominante. Scripta Nova. Revista Electrónica de Geografía y Ciencias Sociales.

Barcelona: Universidad de Barcelona, 15 de Marzo de 2020, vol. XXIV, nº 634. [ISSN: 1138-9788] 